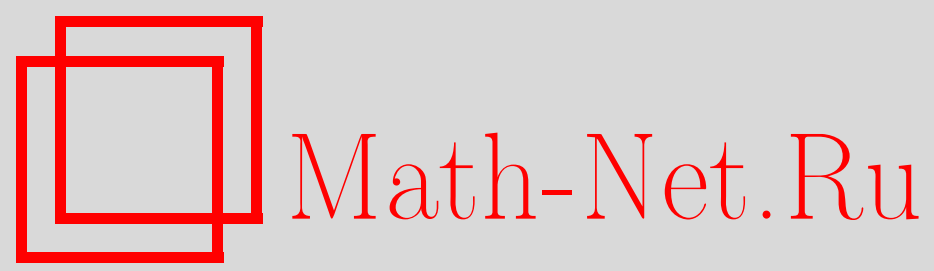

А. И. Михайлов, Функциональная механика: эволюция моментов функции распределения и теорема о возвращении, Вестн. Сам. гос. техн. ун-та. Сер. Физ.мат. науки, 2011, выпуск 1(), 124-133

DOI: https://doi.org/10.14498/vsgtu897

Использование Общероссийского математического портала Math-Net.Ru подразумевает, что вы прочитали и согласны с пользовательским соглашением http://www.mathnet.ru/rus/agreement

Параметры загрузки:

IP: 54.198 .187 .58

26 апреля 2023 г., 04:21:53 


\title{
УДК 517.958 \\ ФУНКЦИОНАЛЬНАЯ МЕХАНИКА: ЭВОЛЮЦИЯ МОМЕНТОВ ФУНКЦИИ РАСПРЕДЕЛЕНИЯ И ТЕОРЕМА О ВОЗВРАЩЕНИИ
}

\author{
А. И. Михайлов \\ 1 Всероссийский научно-исследовательский институт рыбного хозяйства и океанографии, \\ 107140, г. Москва, ул. Верхняя Красносельская, 17. \\ 2 Математический институт им. В.А. Стеклова РАН, \\ 119991, Москва, ул. Губкина, 8. \\ E-mail: mikhailov1984@gmail.ru
}

Рассматривается один из современных подходов к проблеме согласования классической механики и статистической физики - функииональная механика. Вычислены поправки к классическим траекториям и построена эволюиия моментов функиии распределения. Обсуждается связь полученных результатов с отсутствием парадокса Пуанкаре-Цермело в функциональной механике. Показано разрушение периодичности движения в функииональной механике и вычислен декремент затухания для классических инвариантов движения на траектории функиионально-механических средних.

Ключевые слова: классическая механика, проблема необратимости, уравнение Лиувилля.

1. Введение. Проблема необратимости, т. е. вопрос о способе редукции необратимых по времени уравнений макроскопической динамики (уравнений переноса и др.) к фундаментальным микроскопическим уравнениям (уравнениям Гамильтона и Шрёдингера) является одной из наиболее глубоких проблем оснований физической теории. С тех пор как были сформулированы парадоксы Лошмидта и Пуанкаре-Цермело, устанавливающие противоречие между картиной релаксации к термодинамическому равновесию и основополагающими свойствами механического движения, стало ясно, что редукционизм в узком смысле, т.е. вывод макроскопических уравнений только из оснований механики, скорее всего, невозможен, да и методологически неприемлем. Одним из проявлений вышеозначенного противоречия является тот факт, что из теоремы Пуанкаре о возвращении следует невозможность существования энтропии как функции канонических переменных, монотонно возрастающей на траекториях системы. С другой стороны, истинность как уравнений механики, так и уравнений физической кинетики делает необходимым построение процедуры вывода макроскопических уравнений из некоторых дополнительных прескрипций, причём в рамках этих прескрипций вывод должен быть однозначен, а сами прескрипции не зависимы от конкретного вида гамильтониана системы. K настоящему моменту был предложен ряд подходов к решению проблемы необратимости, и тем не менее она всё еще остаётся не исследованной до конца и требует дополнительной проработки. Большинство современных подходов рассматривают свойства макроскопической динамики как атрибуты фазового потока механической системы как целого, а не одной изолированной траектории, существенно различаясь в реализации этого методологического принципа.

Андрей Игоревич Михайлов, младший научный сотрудник, лаб. системного анализа промысловых биоресурсов ${ }^{1}$; младший научный сотрудник, научно-образовательный центр ${ }^{2}$. 
Наиболее известным подходом такого рода является метод Боголюбова [1], заключающийся в построении цепочки уравнений Боголюбова-Борна-Грина-Кирквуда-Ивона путём усреднения многочастичного уравнения Лиувилля. Другой оригинальный метод развит Пригожиным [2]. Этот метод также исходит из уравнения Лиувилля, однако предлагает совсем иную его интерпретацию, основанную на аналогии между уравнениями Лиувилля и Шрёдингера. Скобка Пуассона с функцией Гамильтона (оператор Лиувилля) рассматривается как аналог гамильтониана, действующего в пространстве состояний - функций распределения, а в роли макроскопических величин (прежде всего температуры и энтропии) выступают операторы, не коммутирующие с оператором Лиувилля. Недавно И. В. Воловичем был предложен еще один способ интерпретации уравнения Лиувилля как фундаментального уравнения механики - функциональная механика [3], также рассматривающая в качестве состояния системы функцию распределения, но состояние не макроскопической, а микроскопической системы. Поскольку функциональная механика модифицирует уравнения классической микроскопической динамики, необходимо разобраться, к каким наблюдаемым последствиям приводит подобная модификация. В первой статье (см. [3]), посвященной функциональной механике, было предположено существование отклонений функционально-механических средних от ньютоновских траекторий и в рамках теории возмущений было показано на примере простейших нелинейных механических систем, что такие отклонения имеют место. Ниже будет выведена общая формула для поправок к ньютоновским траекториям в виде разложения по моментам начального распределения и вычислена эволюция всех моментов более высокого порядка. Полученные результаты в дальнейшем использованы для доказательства разрушения периодичности в функциональной механике и несохранения классических инвариантов движения на функционально-механических траекториях. Представленная статья, смею надеяться, позволит подробнее осветить наблюдаемые следствия функциональной механики.

2. Уравнение Лиувилля и эволюция моментов функции распределения. Уравнение Лиувилля

$$
\begin{gathered}
\partial_{t} \rho+\partial_{i}\left(\rho v^{i}\right)=0 \\
\rho(0, x)=\rho_{0}(x)
\end{gathered}
$$

определяет динамику системы в функциональной формулировке классической механики. Здесь $x$ принадлежит фазовому пространству, т. е. является парой переменных $x=(p, q)$, где $p, q \in \mathbb{R}^{n}$, а

$$
\boldsymbol{v}=\sum_{\mu} \frac{\partial H}{\partial p_{\mu}} \partial_{q_{\mu}}-\frac{\partial H}{\partial q_{\mu}} \partial_{p_{\mu}}
$$

задаёт векторное поле, соответствующее гамильтоновой системе с гамильтонианом $H(p, q)$. Состояние механической системы определяется плотностью вероятности $\rho(x)$. Имеет смысл провести параллели с квантовой механикой: плотность вероятности является аналогом матрицы плотности, а линейные функционалы над пространством состояний имеют смысл наблюдаемых. Если в случае квантовой механики наблюдаемые определяются самосопряженными операторами и среднее значение наблюдаемой $\langle\hat{O}\rangle=\operatorname{Tr} \hat{O} \hat{\rho}$, 
то в случае функциональной механики наблюдаемые являются с-числовыми интегрируемыми ${ }^{1}$ по мере $\rho$ функциями и среднее значение наблюдаемой $\langle O\rangle=\int O(x) \rho(x) d x$. Поскольку в функциональной механике наблюдаемые коммутируют друг с другом, проблема одновременного измерения отсутствует - в частности, импульс и координату можно измерить одновременно с любой наперед заданной (но не абсолютной, что существенно) точностью, определяемой свойствами прибора ${ }^{2}$, и именно поэтому функциональная механика является классической.

Решение задачи Коши (1), (2) для уравнения Лиувилля может быть записано в виде $\rho(t, x)=\rho_{0}(u(-t, x))$, где $u(-t, x)$ - фазовый поток вдоль решений уравнений характеристик

$$
\begin{gathered}
\dot{u}(t, x)=v(u), \\
u(0, x)=x,
\end{gathered}
$$

т. е. семейство решений задачи Коши для уравнений динамической системы при при всех возможных начальных данных.

Рассмотрим не только гамильтоновы системы, но и более широкий класс динамических систем с сохраняющим фазовый объём потоком $\operatorname{det}\left(\partial u^{i} / \partial x^{j}\right)=$ $=1$, для которых осмыслена функциональная переформулировка динамики в силу

$$
\int f(x) \rho_{0}(u(-t, x)) d x=\int f(u(t, x)) \rho_{0}(x) d x .
$$

Формально решение задачи (3), (4) может быть представлено экспонентой векторного поля $u(t, x)=e^{t \boldsymbol{v}} x$, где экспонента представляется суммой ряда

$$
e^{t \boldsymbol{v}} \equiv 1+t v^{i} \partial_{i}+\frac{1}{2} t^{2} v^{k} \partial_{k} v^{i} \partial_{i}+\ldots=\sum_{n=0}^{\infty} \frac{t^{n}}{n !}\left(v^{i} \partial_{i}\right)^{n} .
$$

Точное решение не обязательно получать суммированием ряда, оно может быть получено и каким-либо другим способом: суммированием ряда теории возмущений или же в явном виде (в некоторых специальных случаях). Все дальнейшие рассуждения строятся на том предположении, что точное решение известно.

Из теории обыкновенных дифференциальных уравнений известно, что функция $u(t, x)$ непрерывно дифференцируема столько же раз, сколько и $v$,

\footnotetext{
${ }^{1}$ Естественно было бы предположить, что и состояние, и наблюдаемые интегрируемы с квадратом, однако в исходной статье по функциональной механике постановка задачи о поправках к ньютоновским траекториям предполагала расширение пространства состояний до какого-либо пространства обобщенных функций, что, в свою очередь, сузило бы класс наблюдаемых. Хотя в нашем вычислении поправок мы не будем прибегать к аппарату обобщённых функций, следует отметить, что вопрос о классе функций состояния нуждается в дополнительном уточнении.

2 Заметим, что аналог квантово-механической аксиомы измерения в функциональной механике пока еще не сформулирован. Можно лишь утверждать, что, как и в квантовой механике, при измерении происходит редукция функции плотности (см. [4]), однако конкретный вид функции плотности после измерения и его связь со свойствами прибора требует дополнительного обсуждения.
} 
в частности, если $v$ является аналитической, то и $u(t, x)$-аналитическая (см. например [5] для «локальной» теоремы и [6] для «глобальной») на некотором интервале времени, поэтому это решение можно разложить в ряд Тейлора по координате $\xi=x-x_{0}$ вблизи некоторой точки $x_{0}=\int x \rho_{0}(x) d x$, выбранной так, чтобы она совпадала с математических ожиданием координаты по начальному распределению:

$$
u\left(t, x_{0}+\xi\right)=\sum_{\alpha \in \mathbb{N}_{0}^{D}} \frac{1}{\alpha !} \xi^{\alpha} \partial_{\alpha} u\left(t, x_{0}\right) \equiv \sum_{\alpha} \xi^{\alpha} u_{\alpha} .
$$

Здесь использована компактная запись ряда Тейлора через мультииндекс $\alpha \in \mathbb{N}_{0}^{D}$ - вектор с неотрицательными целочисленными компонентами, для которого помимо операций линейной алгебры определены операции взятия модуля $|\alpha|=\sum_{i=1}^{D} \alpha_{i}$, покомпонентного умножения $\alpha \beta=\left\{\alpha_{i} \beta_{i}\right\}$, факториала $\alpha !=\prod_{i=1}^{D} \alpha_{i} !$, а также возведение вектора в степень $x^{\alpha}=\prod_{i=1}^{D} x_{i}^{\alpha_{i}}$ и взятие частной производной $\partial^{\alpha}=\prod_{i=1}^{D} \partial_{i}^{\alpha_{i}}$ порядка $\alpha$. Коэффициент ряда Тейлора, т. е. частная производная порядка $\alpha$ с соответствующим весом обозначена как $u_{\alpha}=u_{\alpha}(t)=\partial^{\alpha} u / \alpha !$.

Теперь, усредняя (5) по начальному распределению, легко вычислить средние значения координат и отклонения средних значений от ньютоновских траекторий

$$
\begin{gathered}
\langle u(t, x)\rangle=\sum_{\alpha} M^{\alpha} u_{\alpha}, \\
\langle u(t, x)\rangle-u\left(t, x_{0}\right)=\sum_{|\alpha|>1} M^{\alpha} u_{\alpha},
\end{gathered}
$$

через центрированные моменты начального распределения $M^{\alpha}$ определяемые

$$
\begin{aligned}
M^{\alpha}=\left\langle\prod_{i=1}^{D}\left(x^{i}-x_{0}^{i}\right)^{\alpha_{i}}\right\rangle=\int \prod_{i=1}^{D}\left(x^{i}-x_{0}^{i}\right)^{\alpha_{i}} \rho_{0}(x) d x= \\
=\int \prod_{i=1}^{D}\left(\xi^{i}\right)^{\alpha_{i}} \rho_{0}\left(x_{0}+\xi\right) d \xi
\end{aligned}
$$

где $\langle u(t, x)\rangle=\int u(t, x) \rho_{0}(x) d x$.

Таким образом, функционально-механические поправки к классическим ньютоновским траекториям заданы счётным набором функций времени $u_{\alpha}-$ всеми частными производными траектории классического движения по начальным данным, вычисленными на самой классической траектории. Поэтому для вычисления отклонений от известной классической траектории нет необходимости находить решение для всех начальных данных - достаточно знать поведение траекторий в окрестности известной или же построить цепочку уравнений в вариациях (см. [6]), задающую эволюцию частных производных решения дифференциального уравнения по параметрам - в данном 
случае начальным данным. После того как искомый набор функций найден, остаётся лишь просуммировать его с заранее определёнными и не меняющимися во времени весами - моментами начального распределения. Видно, что траектория средних значений координат определяется всеми моментами функции распределения в начальный момент времени, следовательно, не существует дифференциального уравнения с конечным числом параметров, которому бы подчинялась траектория средних значений, поскольку решение такого уравнения однозначно определялось бы начальными условиями и параметрами, в то время как траектория средних значений зависит от моментов начального распределения, которые могут быть выбраны достаточно произвольно. Тогда естественным образом возникает задача - проследить эволюцию центрированных моментов всех порядков. Для этого необходимо в исходном определении (8) сделать замену $x \mapsto u(t, x)$ и подставить ряд (6) в новое определение ${ }^{3}$ центрированного момента порядка $\alpha$, а потом переставить операции умножения и суммирования. Проделаем эту громоздкую выкладку в три этапа. Вначале преобразуем центрированный момент с помощью

$$
\begin{array}{r}
M^{\alpha}(t)=\left\langle(u(t, x)-\langle u(t, x)\rangle)^{\alpha}\right\rangle=\left\langle\sum_{\beta \leqslant \alpha}\left((-1)^{|\beta|} u^{\alpha-\beta}(t, x)\langle u(t, x)\rangle\right)^{\beta}\right\rangle= \\
=\sum_{\beta \leqslant \alpha}(-1)^{|\beta|}\left\langle u^{\alpha-\beta}(t, x)\right\rangle\langle u(t, x)\rangle^{\beta},
\end{array}
$$

сделав в исходном определении (8) замену $x \mapsto u(t, x)$. Затем рассчитаем среднее значение монома произвольной степени от координат в некоторый момент времени:

$$
\begin{aligned}
\left\langle u^{\alpha-\beta}(t, x)\right\rangle=\left\langle\left(\sum_{\gamma} \xi^{\gamma} u_{\gamma}\right)^{\alpha-\beta}\right\rangle & = \\
& =\sum_{\gamma}\left\langle\xi^{(\alpha-\beta) \gamma}\right\rangle u_{\gamma}^{\alpha-\beta}=\sum_{\gamma} M^{(\alpha-\beta) \gamma} u_{\gamma}^{\alpha-\beta} .
\end{aligned}
$$

Здесь нижний мультииндекс означает порядок производной, а верхний - степень, в которую нужно возвести её компоненты. Наконец, осталось преобразовать степень среднего значения координат, выделив коэффициенты при произведениях моментов, для чего мы воспользуемся преобразованием

$$
\begin{aligned}
&\langle u(t, x)\rangle^{\beta}=\prod_{i=1}^{D}\left(\sum_{\delta} M^{\delta} u_{\delta}^{i}\right)^{\beta_{i}}= \\
&=\sum_{\substack{\delta_{k} ; \\
\delta_{k} \leqslant \delta_{k+1} \forall k}}\left(\left(\sum_{\substack{\text { перестановки } \\
1 \leqslant i_{k} \leqslant D}} \prod_{\substack{k=1 ; \\
\delta_{k} \leqslant \delta_{k+1} \forall k}}^{|\beta|} u_{\delta_{k}}^{i_{k}}\right) \prod_{\substack{k=1 ; \\
\delta_{k} \leqslant \delta_{k+1} \forall k}}^{|\beta|} M^{\delta_{k}}\right) .
\end{aligned}
$$

${ }^{3} M^{\alpha}(0)=M^{\alpha}$ в силу того, что $u(0, x)=x$. 
Здесь суммирование в коэффициенте перед упорядоченным произведением моментов ведётся таким образом, чтобы перебрались все возможные перестановки целочисленного индекса $i$ так, чтобы индекс $i$-той компоненты появлялся не более чем $\beta_{i}$ раз.

Теперь можно построить алгоритм для вычисления любого момента распределения, если заданы начальные моменты:

$$
\begin{aligned}
& M^{\alpha}(t)=\sum_{\delta_{k} ; \delta_{k} \leqslant \delta_{k+1} \forall k} \sum_{\beta \leqslant \alpha}(-1)^{|\beta|}\left(u_{\gamma}^{\alpha-\beta}\left(\sum_{\substack{\text { перестановки } \\
1 \leqslant i_{k} \leqslant D}} \prod_{\substack{k=1 ; \\
\delta_{k} \leqslant \delta_{k+1} \forall k}}^{|\beta|} u_{\delta_{k}}^{i_{k}}\right)\right) \times \\
& \times\left(M^{(\alpha-\beta) \gamma} \prod_{\substack{k=1 ; \\
\delta_{k} \leqslant \delta_{k+1} \forall k}}^{|\beta|} M^{\delta_{k}}\right) .
\end{aligned}
$$

Заметим, что если распределение имеет вид $\rho(x)=\left(1 / \varepsilon^{D}\right) \phi(x / \varepsilon)$, то для его моментов справедлива следующая оценка по порядку величины: $M^{\alpha} \sim \varepsilon^{|\alpha|}$. Поэтому, используя (10)) в численных расчётах, мы можем удерживать лишь конечное число членов ряда, сохраняя приемлемую точность при исследовании динамики средних значений канонических переменных на временах, сравнимых с периодом.

В качестве примера вычислим матрицу вторых моментов:

$$
\begin{aligned}
& B^{i j}(t)=\left\langle\left(u^{i}(t, x)-\left\langle u^{i}(t, x)\right\rangle\right)\left(u^{j}(t, x)-\left\langle u^{j}(t, x)\right\rangle\right)\right\rangle= \\
= & \left\langle u^{i}(t, x) u^{j}(t, x)\right\rangle-\left\langle u^{j}(t, x)\right\rangle\left\langle u^{j}(t, x)\right\rangle=\sum_{\alpha, \beta} u_{\alpha}^{j} u_{\beta}^{j}\left(M^{\alpha+\beta}-M^{\alpha} M^{\beta}\right) .
\end{aligned}
$$

Первый неисчезающий член в (11) совпадает с выражением

$$
B_{(0)}^{i j}(t)=\sum_{\substack{|\alpha|+|\beta| \geqslant 2 ; \\|\alpha|,|\beta| \leqslant 1}} u_{\alpha}^{j} u_{\beta}^{j} M^{\alpha+\beta}=\frac{\partial u^{i}}{\partial x^{k}} \frac{\partial u^{j}}{\partial x^{l}} B^{k l}
$$

для преобразования тензора второго ранга при замене координат $x \rightarrow u(x)$. Следующая по $\varepsilon$ поправка к (12) задаётся соотношением

$$
\begin{aligned}
B_{(1)}^{i j}(t)=\sum_{\substack{|\alpha|+|\beta| \geqslant 3 ; \\
|\alpha|,|\beta| \leqslant 2}} u_{\alpha}^{j} u_{\beta}^{j}\left(M^{\alpha+\beta}-M^{\alpha} M^{\beta}\right) & \\
=\frac{1}{2}\left(\frac{\partial^{2} u^{i}}{\partial x^{k} \partial x^{m}} \frac{\partial u^{j}}{\partial x^{l}}\right. & \left.+\frac{\partial u^{i}}{\partial x^{k}} \frac{\partial^{2} u^{j}}{\partial x^{m} \partial x^{l}}\right) B^{k m l}+ \\
& +\frac{1}{4} \frac{\partial^{2} u^{i}}{\partial x^{k} \partial x^{m}} \frac{\partial^{2} u^{j}}{\partial x^{l} \partial x^{n}}\left(B^{k m l n}+B^{k m} B^{l n}\right)
\end{aligned}
$$

и содержит моменты более высокого порядка. 
При достаточно малых временах можно воспользоваться разложением экспоненты векторного поля в ряд и получить

$$
\begin{aligned}
& B_{(0)}^{i j}(t) \approx B^{i j}+t\left(\frac{\partial v^{i}}{\partial x^{l}} B^{l j}+\frac{\partial v^{j}}{\partial x^{l}} B^{i l}\right)+ \\
& +\frac{t^{2}}{2}\left(\frac{\partial\left(v^{k} \partial_{k} v^{i}\right)}{\partial x^{l}} B^{l j}+\frac{\partial\left(v^{k} \partial_{k} v^{j}\right)}{\partial x^{l}} B^{i l}\right)+t^{2} \frac{\partial v^{i}}{\partial x^{k}} \frac{\partial v^{j}}{\partial x^{l}} B^{k l} .
\end{aligned}
$$

Выражение (13) воспроизводит явление, аналогичное расплыванию волнового пакета в квантовой механике. Полученные в этом пункте результаты позволяют отслеживать процесс «расплывания» на формально сколь угодно длительных временах - достаточно лишь рассчитать классическую траекторию, начинающуюся в точке фазового пространства, соответствующей математическому ожиданию фазовых переменных по начальному распределению, вычислить частные производные классического решения уравнений движения по начальным данным и задать начальное распределение всеми его моментами (формула (10) описывает эволюцию моментов всех порядков в процессе «расплывания» начального распределения при переносе его фазовым потоком).

3. Заключение. Рассмотрим вопрос применимости полученных результатов. Все рассуждения опирались на две предпосылки: 1) аналитичность фазового потока для достаточно долгого промежутка времени; 2) существование всех конечных моментов функции распределения. «Глобальная» теорема о непрерывной дифференцируемости решения системы (3) по начальным данным, на которую уже имелась ссылка в предыдущем пункте статьи [6], гарантирует сохранение класса гладкости непродолжаемого решения в некоторой его окрестности, поэтому достаточно бесконечной продолжаемости решений системы (3), чтобы обеспечить их аналитичность при аналитичности правой части. Однако, если движение финитно, т. е. траектории, начавшиеся в некоторой области $G$, не покидают её, а векторное поле, задающее правую часть системы (3), определено во всей области $G$ и не имеет в $G$ особых точек, то решения системы (3) с начальными условиями в области $G$ будут бесконечно продолжаемы. Для гамильтоновых систем вышеизложенные условия следуют из компактности поверхности постоянной энергии, некоторую окрестность которой следует выбрать в качестве области $G=\{x \equiv(p, q): h-\delta<$ $<H(p, q)<h+\delta\}$. Тогда аналитичность правой части системы (3) в области $G$ обеспечивает бесконечную дифференцируемость непродолжаемого решения системы (3) по начальным данным и аналитичность $u(t, x)$ в некоторой окрестности $x_{0}$, а значит, выражения (5), (6) и (10) будут применимы на сколь угодно длительных временах, как сравнимых с периодом возвращения, предсказываемого теоремой Пуанкаре, так и превышающих этот период. С другой стороны, существование слабого предела функции распределения (см. [7]) приводит к тому, что всякая траектория средних значений канонических переменных заканчивается в значениях координат средних по предельному распределению, а значит, на бесконечно больших временах, когда нужно учитывать все члены ряда, формула (10) неудобна, поскольку не позволяет выделить асимптотику. Сохранение фазового объёма и финитность движения являются условиями теоремы Пуанкаре о возвращении, однако функ- 
циональная механика предсказывает возникновение отклонений траекторий, усреднённых от начальной точки за период:

$$
\left\langle\sum_{i=1}^{D}\left(u^{i}(t, x)-u^{i}(0, x)\right)^{2}\right\rangle=\sum_{\substack{\alpha, \beta \\ 1 \leqslant|\alpha|,|\beta|}} M^{\alpha+\beta} \sum_{i=1}^{D}\left(u_{\alpha}^{i} u_{\beta}^{i}\right) .
$$

Указанный эффект возникает в силу зависимости периода от начальной точки ${ }^{4}$. Дифференцируя условие периодичности $u(T(x), x)=u(0, x)$ по координате

$$
\partial^{\alpha} u(0, x)=\partial^{\alpha} u(T(x), x)=\prod_{i=1}^{D}\left(\partial_{i}+\partial_{i} T(x)\right)^{\alpha_{i}} u(t, x),
$$

получаем непериодичность старших производных $u_{\alpha}(T(x), x) \neq u_{\alpha}(0, x)$, при этом глобальная периодичность в функциональной механике сохраняется.

Разрушение периодичности в функциональной механике приводит к несохранению на траекториях средних значений координат интегралов движения классической системы. Используя рассуждения, аналогичные вычислению эволюции моментов, можно получить выражение для поправок к эволюции инвариантов классической системы:

$$
\begin{aligned}
\delta I= & I(\langle u(t, x)\rangle)-I(u(t, x))=\sum\left(\sum_{|\alpha| \geqslant 1} u_{\alpha} M^{\alpha}\right)^{\beta} I_{\beta}= \\
& \left.=\sum_{\beta} I_{\beta} \sum_{\substack{1 \leqslant \alpha_{k} ; \\
\alpha_{k} \leqslant \alpha_{k+1} \forall k}}\left(\sum_{\substack{\text { перестановки } \\
1 \leqslant i_{k} \leqslant D}} \prod_{\substack{k=1 ; \\
\alpha_{k} \leqslant \alpha_{k+1} \forall k}}^{|\beta|} u_{\alpha_{k}}^{i_{k}}\right) \prod_{\substack{k=1 ; \\
\alpha_{k} \leqslant \alpha_{k+1} \forall k}}^{|\beta|} M^{\alpha_{k}}\right) .
\end{aligned}
$$

Первый неисчезающий член разложения (14) даётся выражением

$$
\delta I^{(0)}=\frac{1}{2} B^{i j} \frac{\partial^{2} u^{k}}{\partial x_{i} \partial x_{j}} \frac{\partial I}{\partial x_{k}}
$$

и пропорционален матрице ковариаций.

Таким образом, возникает парадокс: в функциональной механике средние значения инвариантов движения, например энергии, сохраняются, но как функции средних значений координат инварианты, и в частности гамильтониан, не сохранятся. Представляется естественным предположить убывание гамильтониана на траектории функционально-механических средних для гамильтоновых систем с компактной и выпуклой поверхностью постоянной энергии, в силу того что $H(\bar{p}, \bar{q})<H(p(t), q(t))$, где $(\bar{p}, \bar{q})$ - усреднённые по времени фазовые переменные $(p(t), q(t))$, а усреднение по слабому пределу вероятностной меры как самих наблюдаемых, так и их временных средних совпадает в силу

\footnotetext{
${ }^{4}$ Понятно, что для точек, лежащих на одной орбите, период одинаков.
} 


$$
\begin{aligned}
& \int\left(\lim _{T \rightarrow+\infty} \frac{1}{T} \int_{0}^{T} u(t, x) d t\right) \bar{\rho}(x) d x=\lim _{T \rightarrow+\infty} \frac{1}{T} \int_{0}^{T} \int u(t, x) \bar{\rho}(x) d x d t= \\
= & \lim _{T \rightarrow+\infty} \frac{1}{T} \int_{0}^{T} \int x \bar{\rho}(u(-t, x)) d x d t=\lim _{T \rightarrow+\infty} \frac{1}{T} \int_{0}^{T} \int x \bar{\rho}(x) d x d t=\int x \bar{\rho}(x) d x .
\end{aligned}
$$

Здесь использована инвариантность слабого предела относительно фазового потока.

Вышеизложенное означает, что предсказание динамики существенно зависит от того, какие величины измеряются. Измерение инвариантов позволяет гораздо точнее предсказать состояние классической системы в последующие моменты времени. Последнее можно интерпретировать двояким образом: однократное измерение инварианта динамической системы эквивалентно многократному измерению фазовых переменных, или, что то же самое, инварианты допускают последовательное измерение, в то время как измерение фазовых переменных должно быть параллельным (одновременным).

Итак, с помощью предложенных в предыдущем пункте формул для эволюции моментов распределения показано, что траектории функциональномеханических средних значений непериодичны и на них не сохраняются инварианты движения, т. е. функциональная механика свободна от парадокса Пуанкаре-Цермело и описывает эффективную диссипацию, которая имеет место в реальных механических системах.

Автор выражает искреннюю признательность И. В. Воловичу и О. Г. Смолянову за постановку задачи, а также благодарит О. В. Грошева, Е. В. Писковского, А. С. Трушечкина и других участников спецсеминара НОЦ МИАН по проблемам необратимости за ценные обсуждения.

Работа выполнена при поддержке Российского фонда фундаментальных исследований (проект № 09-01-12161-офи_м).

\section{БИБЛИОГРАФИЧЕСКИЙ СПИСОК}

1. Боголюбов Н.Н. Проблемы динамической теории в статистической физике. М.-Л.: ОГИЗ. Гостехиздат, 1946. 120 с.; англ. пер.: Bogoluybov N. N. Problems of a dynamical theory in statistical physics / Studies in statistical mechanics. Vol.1. Amsterdam: NorthHolland; New York: Interscience, 1962. 118 pp.

2. Prigogine $I$. From being to becoming: time and complexity in the physical sciences. San Francisco: W. H. Freeman and Co., 1980. 272 pp.; русск. пер.: Пригожин И. От существующего к возникающему: время и сложность в физических науках / Синергетика: от прошлого к будущему / изд. 3-е, доп.; пер. с англ./ ред. Ю. Климонтович. М.: УРСС, 2006. 296 c.

3. Волович И. В. Проблема необратимости и функциональная формулировка классической механики // Вестн. Сам. гос. ун-та. Естественнонаучн. сер., 2008. №8/1(67). C. 35-55, arXiv:0907.2445 [cond-mat.stat-mech]. [Volovich I. V. Time Irreversibility Problem and Functional Formulation of Classical Mechanics // Vestn. Samar. Gos. Univ. Estestvennonauchn. Ser., 2008. no. 8/1(67). Pp. 35-55].

4. Трушечкин A.C. Необратимость и роль измерительного прибора в функциональной формулировке классической механики // TMФ, 2010. Т. 164, № 3. С. 435-440; англ. пер.: Trushechkin A.S. Irreversibility and the role of an instrument in the functional formulation of classical mechanics // Theoret. and Math. Phys., 2010. Vol.164, no. 3. Pp. 1198-1201.

5. Тихонов А.Н., Васильева А.Б., Свешников А.Г. Дифференциальные уравнения. М.: Физматлит, 2005. 256 с.; англ. пер.: Tikhonov A.N., Vasil'eva A.B., Sveshnikov A.G. Differential equations. Berlin etc.: Springer-Verlag, 1985. 238 pp. 
6. Понтрягин Л. С. Обыкновенные дифференциальные уравнения. М.: Наука, 1982. 332 с.; англ. пер.: Pontryagin L. S. Ordinary Differential Equations. London: Addison-Wesley, 1962. 298 pp.

7. Козлов В.В. Тепловое равновесие по Гиббсу и Пуанкаре. М., Ижевск: Институт компьютерных исследований, 2002. 320 с. [Kozlov $V$. V. Thermal equilibrium in the sense of Gibbs and Poincaré. Moscow, Izhevsk: Institut Komp'yuternykh Issledovanij, 2002. 320 pp.]

MSC: 82C05

\title{
THE FUNCTIONAL MECHANICS: EVOLUTION OF THE MOMENTS OF DISTRIBUTION FUNCTION AND THE POINCARÉ RECURRENCE THEOREM
}

\author{
A. I Mikhailov ${ }^{1,2}$ \\ 1 Russian Federal Research Institute of Fisheries and Oceanography, \\ 17, Verkhnyaya Krasnosel'skaya st., Moscow, 107140, Russia. \\ 2 Steklov Mathematical Institute, Russian Academy of Sciences, \\ 8, Gubkina st., Moscow, 119991, Russia. \\ E-mail: mikhailov1984@gmail.ru
}

One of modern approaches to a problem of the coordination of classical mechanics and the statistical physics - the functional mechanics is considered. Deviations from classical trajectories are calculated and evolution of the moments of distribution function is constructed. The relation between the received results and absence of paradox of Poincaré-Zermelo in the functional mechanics is discussed. Destruction of periodicity of movement in the functional mechanics is shown and decrement of attenuation for classical invariants of movement on a trajectory of functional mechanical averages is calculated.

Key words: classical mechanics, irreversibility problem, Liouville equation.

Original article submitted 21/12/2010; revision submitted 15/III/2011.

Andrey I. Mikhailov, Junior Researcher, Lab. of Bioresource Systems Analysis ${ }^{1}$; Junior Researcher, Research and Education Center ${ }^{2}$. 